\title{
Psychological Constructs Influencing Exercise Behavior in Women According to the Transtheoretical Model: A Questionnaire Survey
}

\author{
Asiyeh Pirzadeh, ${ }^{1}$ and Firoozeh Mostafavi ${ }^{1,}$ \\ ${ }^{1}$ Department of Health Education and Promotion, School of Health, Isfahan University of Medical Sciences, Isfahan, IR Iran \\ "Corresponding author: Firoozeh Mostafavi, Department of Health Education and Promotion, School of Health, Isfahan University of Medical Sciences, Isfahan, IR Iran. Tel: \\ +98-3137923201, E-mail: f_mostafavi@yahoo.com
}

Received 2015 February 08; Revised 2016 February 28; Accepted 2016 August 21.

Keywords: Behavior, Exercise, Physical activity

\section{Dear Editor,}

The present cross-sectional study involved 400 women who were referred to a health center in Isfahan, Iran, in 2012. After informed consent was obtained from each individual, a multi-part questionnaire was completed by selfreport.

- The Stages of Exercise Change Questionnaire (SECQ) divided the participants into five stages: precontemplation, contemplation, preparation, action, and maintenance $(\alpha=0.91)$.

- The processes of change questionnaire included 30 items for the measurement of cognitive and behavioral processes ( $\alpha=0.87$ ).

- The decisional balance questionnaire included ten items that were rated on a five-point scale (from $1=$ extremely important to $5=$ not important $)(\alpha=0.78)$.

- The self-efficacy questionnaire included six items that were rated on a five-point scale (from $1=$ not confident to $5=$ very confident $)(\alpha=0.81)$.

- The Godin Leisure-Time exercise questionnaire measured weekly exercise behavior (1).

According to the results of this study, $24.2 \%$ of participants were in the pre-contemplation stage, $33.2 \%$ in the contemplating stage, $25.5 \%$ in the preparation stage, $7.2 \%$ in the action stage, and $9.8 \%$ in the maintenance stage.

Table 1 displays the means of all the variables in the transtheoretical model(TTM). The total physical activity increased from the pre-contemplation stage to the maintenance stage. The ANOVA showed that there were statistically significant differences between the stages $(f=139.98$, $\mathrm{P} \leq 0.001)$. In the present study, most participants were in the inactive stages (i.e., pre-contemplation, contemplation, and preparation), which is consistent with the findings of Lee et al.'s study (2). Applying the processes of change can help people to change their behavior and move on to higher stages (stages of change). However, in the present study, the "social liberation" and "helping relation- ship" factors did not change significantly during the different stages of change, which might be related to the Iranian culture. Hence, educational programs should focus on the TTM constructs that influence the physical activity. Importantly, our previous study revealed that the TTM could be a suitable model for educating women (3).

Unfortunately, statistics show that only a small percentage of the population in many countries is active, while a large percentage of the population does not engage in sufficient physical activity $(4,5)$, especially women. In Iran, the general prevalence of physical inactivity was found to be $69.8 \%$, and only $30.3 \%$ of women took part in adequate physical activity (6).

Today, it is common for a theory or model to be used to identify the factors that influence behavior. The transtheoretical model includes four constructs (stages of change, processes of change, decisional balance, and self-efficacy) that help to identify the psychosocial factors that influence physical activity. Therefore, determining the psychological constructs that influence exercise behavior in women using the transtheoretical model might prove important when designing educational interventions.

The inclusion of only women who were referred to the health center and the self-report questionnaire methodology represent the limitations of this study.

\section{Conclusion}

This study revealed comprehensive information about the factors that affect women's physical activity based on the transtheoretical model, which should help health educators to design effective educational interventions to increase women's physical activity.

\section{Acknowledgments}

We wish to thank all the women who participated in this study. 
Table 1. Mean and Standard Deviation (SD) of the Transtheoretical Model Variables During the Stages of Change ${ }^{\mathrm{a}}$

\begin{tabular}{|c|c|c|c|c|c|c|}
\hline TTM Variables & Pre-Contemplation & Contemplation & Preparation & Action & Maintenance & P Value $(F)$ \\
\hline Consciousness raising & $2.64 \pm 6.19$ & $2.77 \pm 7.27$ & $2.56 \pm 7.73$ & $2.56 \pm 8.65$ & $9.70 \pm 3.14$ & $0.005(4.235)$ \\
\hline Dramatic relief & $2.95 \pm 9.93$ & $3.08 \pm 10.25$ & $2.63 \pm 11.01$ & $2.45 \pm 10.65$ & $11.85 \pm 1.81$ & $0.002(4.512)$ \\
\hline Environmental reevaluation & $2.20 \pm 6.15$ & $2.18 \pm 6.35$ & $1.95 \pm 7.02$ & $1.71 \pm 7.17$ & $8.60 \pm 1.67$ & $<0.001(5.612)$ \\
\hline Self-reevaluation & $11.70 \pm 3.58$ & $12.06 \pm 2.90$ & $12.64 \pm 2.98$ & $13.37 \pm 1.98$ & $14.20 \pm 1.28$ & $<0.001(5.256)$ \\
\hline Social liberation & $11.10 \pm 3.23$ & $10.69 \pm 2.92$ & $10.79 \pm 2.72$ & $11.48 \pm 2.33$ & $11.90 \pm 2.48$ & $0.144(1.856)$ \\
\hline Counter conditioning & $3.18 \pm 6.72$ & $2.87 \pm 7.09$ & $2.72 \pm 7.09$ & $2.70 \pm 9.34$ & $2.40 \pm 9.62$ & $<0.001(5.985)$ \\
\hline Helping relationship & $2.38 \pm 4.63$ & $2.32 \pm 5.08$ & $2.12 \pm 4.67$ & $2.36 \pm 5.82$ & $2.17 \pm 5.22$ & $0.073(1.255)$ \\
\hline Reinforcement management & $3.41 \pm 9.91$ & $2.92 \pm 10.74$ & $2.82 \pm 10.87$ & $2.31 \pm 12.17$ & $2.09 \pm 13.05$ & $<0.001(4.314)$ \\
\hline Stimulus control & $3.63 \pm 6.87$ & $3.06 \pm 7.14$ & $3.06 \pm 7.08$ & $3.16 \pm 9.24$ & $3.53 \pm 10.85$ & $<0.001(4.632)$ \\
\hline Self-liberation & $3.43 \pm 8.79$ & $2.97 \pm 9.19$ & $2.65 \pm 9.46$ & $2.54 \pm 11.10$ & $2.79 \pm 12.05$ & $<0.001(5.314)$ \\
\hline Pros & $4.72 \pm 16.37$ & $4.23 \pm 17.89$ & $4.64 \pm 18.42$ & $4.70 \pm 18.93$ & $20.100 \pm 4.31$ & $<0.001(2.814)$ \\
\hline Cons & $3.87 \pm 18.69$ & $3.98 \pm 18.89$ & $3.74 \pm 18.73$ & $4.57 \pm 18.55$ & $18.82 \pm 3.73$ & $0.991(0.021)$ \\
\hline Self-efficacy & $4.23 \pm 10.78$ & $4.03 \pm 11.24$ & $3.40 \pm 11.45$ & $4.90 \pm 13.03$ & $4.97 \pm 15.17$ & $<0.001(3.214)$ \\
\hline
\end{tabular}

${ }^{\mathrm{a}}$ Data are presented as Mean $\pm \mathrm{SD}$.

\section{Footnote}

Authors' Contribution: Both authors participated in all stages of the study.

\section{References}

1. Godin G, Shephard RJ. A simple method to assess exercise behavior in the community. Can J Appl Sport Sci. 1985;10(3):141-6. [PubMed: 4053261].

2. Lee YM, Park NH, Kim YH. Process of change, decisional balance, self-efficacy and depression across the stages of change for exercise among middle aged women in Korea. Taehan Kanho Hakhoe Chi. 2006;36(4):587-95. [PubMed: 16825842].
3. Pirzadeh A, Mostafavi F, Ghofranipour F, Feizi A. Applying Transtheoretical Model to Promote Physical Activities Among Women. Iran J Psychiatry Behav Sci. 2015;9(4):1580. doi: 10.17795/ijpbs-1580. [PubMed: 26834796]

4. Del Duca GF, Nahas MV, Garcia LM, Mota J, Hallal PC, Peres MA Prevalence and sociodemographic correlates of all domains of physical activity in Brazilian adults. Prev Med. 2013;56(2):99-102. doi: 10.1016/j.ypmed.2012.11.007. [PubMed: 23200875].

5. Mori K, Suzuki H, Wang DH, Takaki J, Takigawa T, Ogino K. Relationship of psychological factors with physical activity stage of change in prime-and middle-aged Japanese. Acta Med Okayama. 2009;63(2):97104. [PubMed: 19404341].

6. Momenan AA, Delshad M, Mirmiran P, Ghanbarian A, Azizi F. Leisure Time Physical Activity and Its Determinants among Adults in Tehran: Tehran Lipid and Glucose Study. Int J Prev Med. 2011;2(4):243-51. [PubMed: 22174964]. 